Editorial

\title{
Sustainable Governance in Northeast Asia: Challenges for the Sustainable Frontier
}

\author{
Yongrok Choi
}

Department of International Trade and Regional Studies, Inha University, 100 Inha-ro, Nam-gu, Incheon 402-751, Korea; yrchoi@inha.ac.kr; Tel.: +82-32-860-7760; Fax: +82-32-876-9328

Academic Editor: Marc A. Rosen

Received: 12 January 2017; Accepted: 20 January 2017; Published: 28 January 2017

\begin{abstract}
This Special Issue consists of selected papers from the annual international Sustainable Asia Conference (SAC) 2016, held on Jeju Island, South Korea, from 28 June to 2 July 2016. SAC 2016 is one of the leading international conferences for presenting novel and fundamental advances in sustainable development issues for Asia. In this special issue, most of the papers emphasize the importance of sustainable governance in harmonizing economic development with a healthier life, while enhancing the quality of all economic activities. The majority of papers in this special issue also deal with problems of urbanization, because the Northeast Asian countries are experiencing the transformation of their economic structure from quantitatively oriented development to the qualitative, highlighting socioeconomic performance. There has been a vast amount of discussion and many ideas put forward on sustainable development, but as the Marrakech Proclamation of COP 22 concluded in November 2016, it is now time to meet the practical challenges of sustainable development. This special edition will shed light on the action plan against global warming and environmental degradation.
\end{abstract}

Keywords: Sustainable Asia Conference (SAC); Post-2020 climate regime; green growth; sustainable governance

\section{Background of the Special Issue}

The Sustainable Asia Conference (SAC) is an academic network for Northeast Asian professors and researchers to share ideas and cooperate in facing new challenges in the field of sustainable development, green growth, and green IT. SAC has been based on its annual international conference since its inaugural event in 2009 at Incheon, Korea. Since then, there have been more than 100 papers published through the conference, and this Special Issue documents one of these annual conferences-SAC 2016, held on Jeju Island, Korea, during 28-29 June 2016. This conference provides opportunities for researchers, experts, and practitioners to exchange new ideas and experiences to find global partners for future collaborations. The scope of this special issue encompasses topics related to sustainable governance in Northeast Asia with challenges for the sustainable frontier.

Northeast Asian countries have witnessed the world's most rapid economic growth, as well as the resulting pollution, such as carbon dioxide $\left(\mathrm{CO}_{2}\right)$ emissions. To cope with these undesirable outcomes, all of the governments in the region have shifted their policy paradigms to place much more emphasis on environmental issues [1]. Due to the ever-increasing complexity of the issues in this field of sustainable development or green growth, there were many diverse views and suggestions put forward in SAC 2016, with most of them focused on matters of governance or the determining factors of various governance mechanisms. The Chinese government is facing severe environmental problems-especially in the winter season every year-due to the ever-increasing smog of particulate matter known as PM 2.5. Thousands of people are suffering from this toxic air pollution, and it has 
become much more severe over time. In particular, this air pollution easily reaches Korea and Japan during the winter due to the cold seasonal northwest wind in the region. This is why international cooperation to overcome this environmental disaster is so crucial in the region.

Korea has also experienced an annual temperature increase higher than any other country in the world, due to its specific environmental situation as a peninsula, resulting in the nation's highest temperature record being surpassed almost every year. Through the strong support of ex-president Lee, Korea hosted the Green Climate Fund, and has strongly promoted green growth policies, including the historical inauguration of its nationwide emission-trading scheme (ETS) in 2015. As the successful host of the Kyoto Protocol, Japan has been strongly prompted to mitigate its greenhouse gas (GHG) emission levels. Unfortunately, other leading countries such as the United States and China did not participate in the Kyoto Protocol, and thus it could not be fully implemented and sustained. Nonetheless, the Kyoto Protocol provided a great platform to deal with environmental issues via sustainable market-oriented solutions with flexible mechanisms, such as ETS, the Clean Development Mechanism, and Joint Implementation. The flexibility of the Kyoto Protocol's mechanisms opened a new frontier in sustainable development, because air pollution at least became measurable, reportable, and verifiable in order to mitigate its undesirable effects on the global economy.

All these Northeast Asian countries share the common goal of green growth. Environmental protection is important, but at the same time, economic development is crucial in a region that has become the engine of global economic growth. Fortunately, green growth policies could create a decoupling of economic development from environmental issues. In particular, the Paris Agreement in 2015 at the COP 21 meeting put in place an important cornerstone to promote the better performance of green growth policies for all these countries from a governance perspective.

Many of the papers presented in SAC 2016 are relevant to this new frontier of the Paris Agreement, and thus this special issue with selected papers from the conference could provide a new platform to implement sustainable governance in the region.

\section{Fresh Challenges under the New 2020 Climate Regime}

While the Kyoto Protocol regime provided a very important stepping-stone toward the marketability of air pollution issues, it lacked governance authority due to the presence of too many passive participants in the mitigation of GHG emissions. Only 38 Annex I developed countries and the European Union committed to mitigate six GHG emissions. This ambitious commitment, however, covered only 22 percent of global emissions, and most countries participating in the protocol are just passive members aiming only to get benefits from this protocol. This certainly resulted in only partially successful operation for the first round of five years from 2008 until 2012. The United States could not ratify the Kyoto protocol due to its market-oriented policy paradigm, and thus many other countries did not participate in the second round of the Kyoto Protocol. In order to mitigate the worsening conditions of the regime, 197 member countries of the UN Framework Convention on Climate Change held their 21st Conference of the Parties (COP 21) in Paris, and agreed to inaugurate the new 2020 climate regime.

Without global efforts, GHG emission levels may reach up to 700 ppm by 2030 with the business as usual (BAU) trajectory shown in Figure 1. At the COP 21 meeting at Paris, the member countries agreed that global warming is not a special issue for some countries, but is the responsibility of all; and that there should be no exceptions among nations in taking steps to mitigate GHG emissions worldwide to achieve below $2{ }^{\circ} \mathrm{C}\left(3.8^{\circ} \mathrm{F}\right)$ from BAU. In order to decrease the average temperature of the Earth, all countries should make efforts to decrease overall emissions by at least 38 gigatons carbon dioxide equivalent $\left(\mathrm{Gt} \mathrm{CO}_{2} \mathrm{e}\right)$ per year by the target year of 2030. COP 21 agreed that-based on all possible technical measures up to 2030-we could and should make our best efforts to decrease emissions by $38 \mathrm{Gt} \mathrm{CO}_{2} \mathrm{e}$. However, it is never easy and effective to use all these potential technical measures, due to the budgets needed to develop and utilize them [2]. These potential technical measures are shown in Figure 2. In this figure, the height of the bar indicating each new technology 
represents the cost or budget for the research and development of this technology, while the width of the bar for each individual technology indicates the effect of GHG emission mitigation [2]. As shown in Figure 2, all these technological measures combined may contribute up to the $38 \mathrm{Gt} \mathrm{CO}_{2}$ e mitigation of GHG emissions.

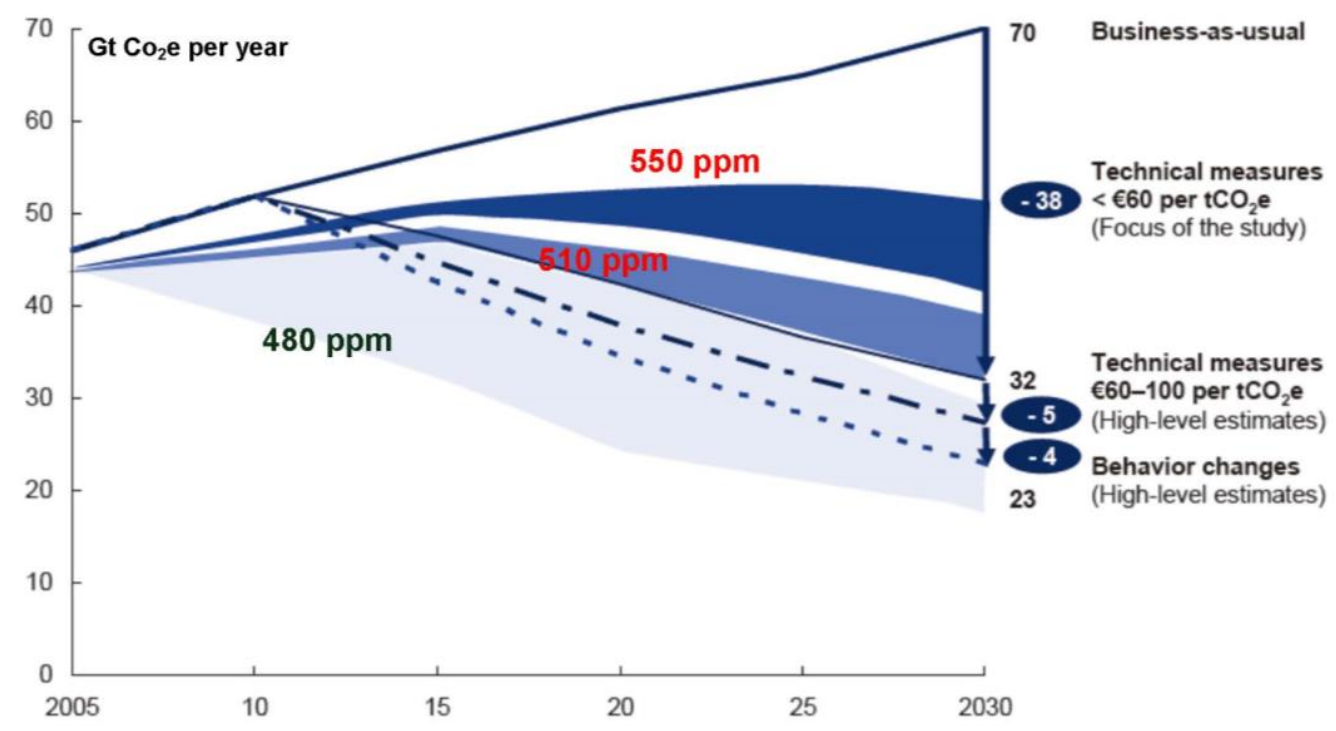

Figure 1. Global greenhouse gas (GHG) Emission Pathways. Source: Global GHG Cost Curve V2.0 (McKinsey \& Co. 2009, [2]); IEA; IPCC; OECD; US EPA.

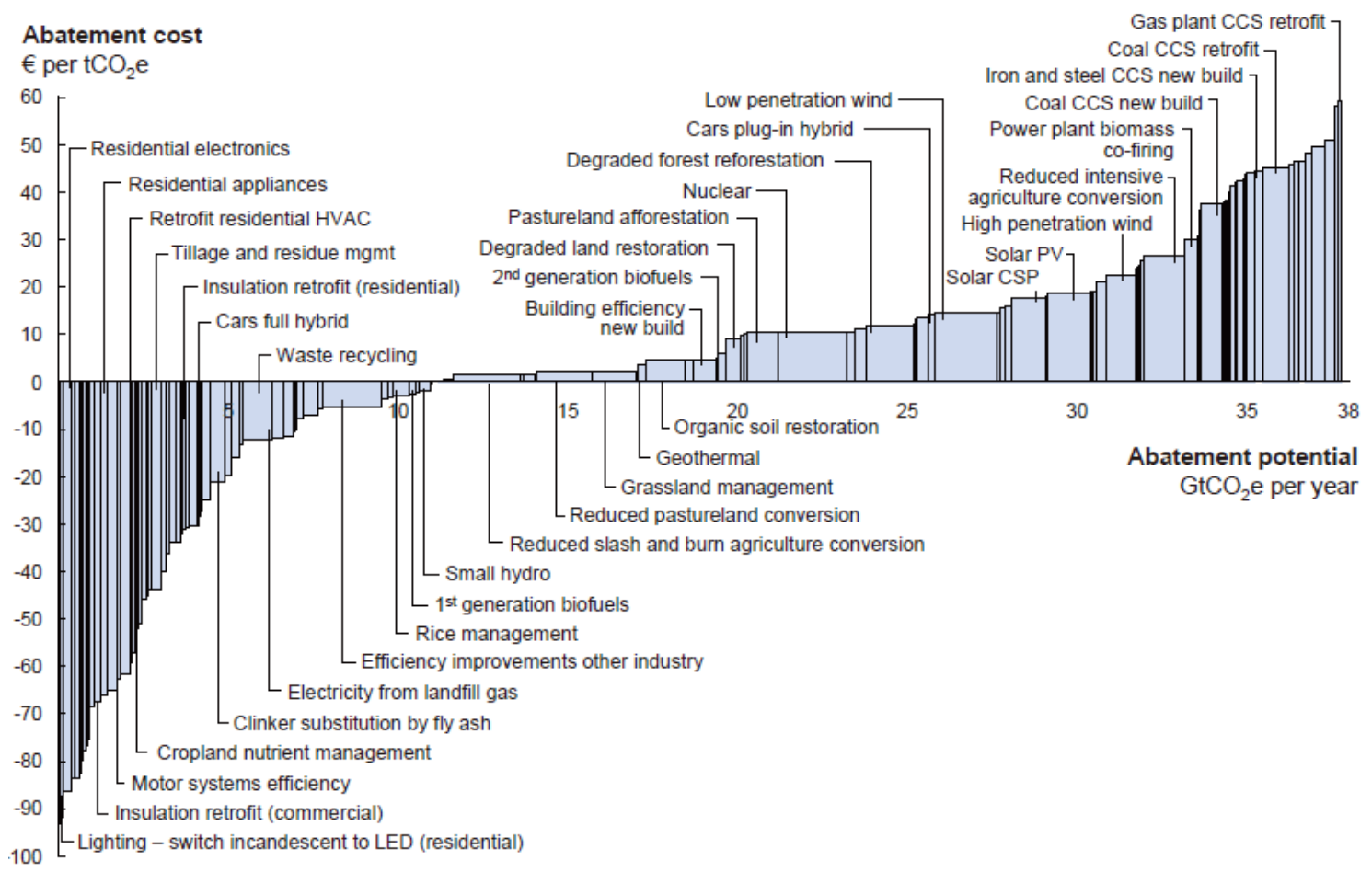

Figure 2. Greenhouse Gas Abatement Opportunities. Source: Global GHG Cost Curve V2.0 (McKinsey \& Co. 2009, [2]). CCS: carbon capture and storage.

Some technologies—such as LED lights in residential areas-may not incur any cost to utilize; over time, they may give additional benefits (negative costs) to households. These technologies could be utilized easily and effectively if governments provided subsidies and/or other incentives to develop 
them, while some more advanced technologies, such as those for carbon capture and storage (CCS), are too risky in their utilization budgets, and thus strong support from public-private partnerships is

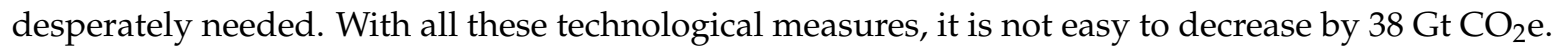
In order to decrease by $1 \mathrm{Gt} \mathrm{CO}_{2} \mathrm{e}$, for example, all the vehicles in the EU would have to be kept from driving for a year [2]. All of these optimal controls on the GHG emission pathways are based on an estimated global GHG emissions peak of 480 ppm by the year 2015 and decreasing thereafter. Unfortunately, time really does matter for this emission pathway, because the optimal path is already mission impossible due to the late response from the COP member countries; thus, we may not have this pathway anymore, and to get the same result for the global temperature, we may need an additional $9 \mathrm{Gt} \mathrm{CO}_{2}$ e reduction through behavioral changes in people. Therefore, the member countries at the COP 22 meeting of 2016 emphasized the gap in the global goal between its mission and the possible efforts of the Marrakech Proclamation. The question is how we are going to fill this gap or missing link in terms of governance and workable mechanisms for sustainable performance [3]. At SAC 2016, many papers addressed the new challenges of governance raised by the 2020 climate regime, and we shall introduce selected papers on this theme from SAC 2016 in this special issue.

\section{Governance Issues for the Sustainability of Asia}

All the papers in this special issue are based on the common paradigm of governance aimed toward green growth. In particular, many papers argue that urbanization is a crucial dimension of policies for green growth, because urbanization was the one of the key successful phenomena in the rapidly growing Northeast Asian countries, although urbanization may face its limits for inclusive, harmonized, and sustainable development. Therefore, appropriate approaches to overcoming the diverse issues of urbanization could provide very basic stepping-stones for governments in implementing the new 2020 climate regime.

Urbanization could be considered as a primary source of the degradation of natural, open ecosystems, and unfortunately, most of this conversion from open land to built-up areas results in significant and irreversible natural habitat loss. To evaluate the ecological changes appropriate to Jiangsu's coastal wetland, Caiyao Xu et al. analyzed ecological security and ecosystem services in the region by using remote sensing and spatial analyses for the period from 1977 to 2014 [4]. They found that the total ecosystem service value decreased significantly from $\$ 2.98$ billion per year to $\$ 2.31$ billion per year over the study period due to this transformation from open to built-up land. Moreover, it will take 34 years for landscape ecological security and 39 years for ecosystem services to regain their original state of equilibrium. Nonetheless, food production was the only ecosystem service function that consistently increased, mainly because of government policy, implying that government regulation or incentives regarding the transformation and/or utilization of the land could be the best solution for green growth in the region [4].

Of course, rapid urbanization stimulated the expansion of industrial production and increased industrial pollutant emissions-especially in China and other developing countries. However, urbanization results in undesirable drawbacks, not only through environmental degradation, but also in unavoidable industrial structure. To remedy this drawback, Jin Guo et al. analyzed the influence of urbanization impacts on industrial pollutant emissions, looking at scale effects, intensive effects, and structural effects, using the Kaya Identity and the Log Mean Divisia Index (LMDI) method based on a spatial panel model [5]. Using data from 282 prefecture-level cities in China from 2003 to 2014, they found a significant inverse U-shape between China's urbanization rate and environmental degradation indicators such as the volume of industrial wastewater discharge and emissions of sulfur dioxide and PM (dust). With the consistent rise in the urbanization rate, the volume of industrial wastewater discharges increased steadily, and reached its peak in 2007. After that, the volume of industrial wastewater discharge showed a downward trend. This clearly shows that the paradigm shift of the Chinese government from the year 2005-with a greater focus on environmental regulations and promotion incentives-could effectively impede the rate of environmental degradation. Moreover, it is 
noteworthy from the findings that diverse surrounding suburban areas of China's cities may not serve as sponge belts absorbing industrial pollutants, because the spatial spillover effect from the central cities to these suburban areas is non-existent or non-significant, implying that a selective concentration on environmental regulation could be much more effective than a wide range of universal treatment [5].

As one of issues related to urbanization, more attention needs to be paid to the efficient use of forested land; thus, Yafen He et al. analyzed the forested land use efficiency (FLUE) and its spatiotemporal differences in China during the period from 1999 to 2010, using a global generalized directional distance function and global Malmquist-Luenberger index models [6]. They found obvious spatial differences in forested land use efficiency among 31 provinces, with Shanghai the best and Tibet, Inner Mongolia, and Qinghai the worst. Since this difference comes from a very complicated mixture of urbanization, economic development contexts, and population density (as shown in the model), it is not easy and effective for the central government to mitigate these differences. To mitigate this gap, Yafen He et al. tried to examine the dynamic changes in the FLUE, and found that the productivity of forested land was always increasing, and that changes in the productivity of forested land in the eastern region derived mostly from technological advances [6]. For the central region, the progress in forested land productivity derived mostly from improvements in efficiency. The change in the productivity of forested land in the western region resulted from the interactive effect of these two decompositions [6]. These findings imply that environmentally friendly green growth policies should be managed differently depending on the unique conditions of regional economic activities and their environmental settings.

As shown in Figures 1 and 2, the mitigation of GHG emissions is a truly important mission for all humankind. However, the Paris Agreement also emphasizes the importance of supportive efforts to enhance eco-efficiency. In this perspective, Shujing Yue et al. analyzed the total-factor ecological efficiency (TFEcE) of G20 countries during the period 1999-2013 using the slack-based Data Envelope Analysis (DEA) model [7]. In contrast with the traditional approach, they used the ecological footprint as one of the inputs, this being a simple assessment for an aggregate area of the productive lands and water required to generate the region's GDP. They found that the average TFEcE of the G20 nations is at a low level of about 0.54 , which definitely needs to be enhanced. This finding provides background support for the new 2020 climate regime for all countries. In particular, China improved throughout the experimental period from 0.238 to 0.285 , with much potential enhancement ahead. Korea shows a value of about 0.7 -much smaller than its potential increase, but with an aggravating trend in efficiency; this also definitely needs to be improved. In contrast with these two countries, Japan is shown to be the best on its productivity frontier for the whole experimental period, indicating the most effective results of the Kyoto Protocol on its economic performance [7]. From these findings, it is extremely noteworthy that technological measures are important in mitigating GHG emissions, while the emphasis on ecological efficiency should not be discounted, because there is huge potential to enhance eco-friendly efficiency.

There are also some conflicts between the higher productivity of agricultural production and the higher quality of its products in terms of organic eco-production systems. Unfortunately, most Chinese farmers tend to use excessive pesticides for higher production, causing moral hazard behavior on food health. Zhang and Li examined how Chinese farmers' moral hazard behavior in crop production is influenced by their traditional culture using a semi-parametric logistic model, and found that Chinese traditional culture has a positive effect on restricting the farmers' excessive use of pesticides in crop production [8]. The probability of moral hazard could decrease by $17 \%$ if farmers become aware of traditional culture as providing a restraint on the use of pesticides. It is noteworthy that even if traditional culture is important to deal with moral hazard on the excessive use of pesticides, formal institutions are more effective in constraining farmers' production behavior, which helps to reduce the probability of moral hazard, implying that the government should implement relevant policies, such as improving the frequency of random inspections, intensifying penalties, and strengthening market 
supervision for agricultural inputs, to evoke culturally conditioned cognitive behavior on the use of pesticides [8].

In a step toward the ambitious green growth pathway under the new climate 2020 regime, Korea just inaugurated a nationwide emissions trading scheme (ETS) in 2015. To establish the feasibility of this ETS policy, Choi and Lee analyzed its sustainable governance factors in terms of carbon technical efficiency, the shadow price of carbon emissions, and Morishima elasticity for green investment, using the non-radial directional distance function [9]. Unfortunately, they found that the market price of carbon emissions is far too low compared with its shadow price, suggesting that the Korean government's price-oriented market intervention has resulted in poor performance, at least in its initial stage [9].

Based on all these papers in this special issue, the Northeast Asian countries are seen to emphasize their harmonized interrelationship in order to enhance sustainable governance in the region. Even if the new climate 2020 regime focuses on efforts toward the mitigation of GHG emissions, most of these papers argue that eco-friendly efficiency could be a new source of green growth. This approach may create fewer budget demands than other technical measures require for their development and utilization; thus, enhancing efforts for efficiency toward more eco-friendly green growth could be a viable alternative for the new 2020 climate regime, especially in developing countries such as China and Korea [10]. This special issue highlights harmonizing these efforts with legacy systems instead of expensive, risky investments on green technology.

\section{Conclusions}

The research contents and methodologies examined in this special issue give us greater insights and open new frontiers to handle the contemporary challenges of sustainable governance. Most of these articles handle the multiple inputs and outputs with time and space in consideration. This emphasizes that sustainable governance requires complex procedural approaches in order to grasp workable mechanisms to harmonize all the interest groups in the cooperative network. Clarifying the causal relations in this network is not easy, but it is essential for the network manager or agency to ensure that all parties share values and face the challenges together. This is the catchphrase of the Sustainable Asia Conference (SAC): Once a friend, forever a friend.

Networking participants striving for harmonized and sustainable cooperative performance will need to adopt a more field- and performance-oriented approach to create these invisible but precious values-this is true sustainable governance [11]. In order to discover these sustainable governance factors, relevant issues and their methodologies should be highlighted in this rapidly-evolving Asian model. In particular, compared with the efficiency-oriented Western countries, the Northeast Asian countries have placed more emphasis on their harmonized interrelationship [11]. This social relationship is absolutely crucial in creating and sharing values in these countries. The world's priorities are presently changing from visible profits and efficiency toward these invisible values, which derive from sustainable cooperative networks in business, in the economy, and in society as a whole. As shown in the diverse perspectives on urbanization and other related issues in this special edition, we should work together to find the appropriate Asian models for this sustainable cooperation network mechanism in the future, especially for SAC 2017 in Nanjing in China on 24 June 2017.

Acknowledgments: The National Research Foundation of Korea Grant funded by the Korean Government (NRF-2014S1A5B1011422) supported this work.

Conflicts of Interest: The author declares no conflict of interest.

\section{References}

1. Choi, Y. Intermediary Propositions for Green Growth with Sustainable Governance. Sustainability 2015, 7 , 14785-14801. [CrossRef] 
2. McKinsey \& Co, Pathways to a Low-Carbon Economy: Version 2 of the Global Greenhouse Gas Abatement Cost Curve, 2009. Available online: http:/ / www.google.co.kr/url?sa=t\&rct=j\&q=\&esrc=s\&source=web\&cd=2\& ved=0ahUKEwjJkK-496TRAhVBW5QKHUZSDdkQFggnMAE\&url=http\%3A\%2F\%2Fwww.mckinsey.com\% 2F \%2Fmedia\%2Fmckinsey\%2Fdotcom\%2Fclient_service\%2Fsustainability\%2Fcost $\% 2520$ curve $\% 2520$ pdfs $\%$ 2F_pathways_lowcarbon_economy_version2.ashx\&usg=AFQjCNHTmNTNQaoqigDxjPymDtYibovpuA (accessed on 25 December 2016).

3. Choi, Y.; Bone, C.; Zhang, N. Sustainable Policies and Strategies in Asia: Challenges for Green Growth. Technol. Forecasti. Soc. Chang. 2016, 112, 135. [CrossRef]

4. Xu, C.; Pu, L.; Zhu, M.; Li, J.; Chen, X.; Wang, X.; Xie, X. Ecological Security and Ecosystem Services in Response to Land Use Change in the Coastal Area of Jiangsu, China. Sustainability 2016, 8, 816. [CrossRef]

5. Guo, J.; Xu, Y.; Pu, Z. Urbanization and Its Effects on Industrial Pollutant Emissions: An Empirical Study of a Chinese Case with the Spatial Panel Model. Sustainability 2016, 8, 812. [CrossRef]

6. He, Y.; Xie, H.; Fan, Y.; Wang, W.; Xie, X. Forested Land Use Efficiency in China: Spatiotemporal Patterns and Influencing Factors from 1999 to 2010. Sustainability 2016, 8, 772. [CrossRef]

7. Yue, S.; Yang, Y.; Shao, J.; Zhu, Y. International Comparison of Total Factor Ecology Efficiency: Focused on G20 from 1999-2013. Sustainability 2016, 8, 1129. [CrossRef]

8. Zhang, L.; Li, X. The Impact of Traditional Culture on Farmers' Moral Hazard Behavior in Crop Production: Evidence from China. Sustainability 2016, 8, 643. [CrossRef]

9. Choi, Y.; Lee, H.S. Are Emissions Trading Policies Sustainable? A Study of the Petrochemical Industry in Korea. Sustainability 2016, 8, 1110. [CrossRef]

10. Choi, Y.; Song, M.; Myeong, S. Introduction to the Special Issue on the Sustainable Asia Conference 2015. Sustainability 2016, 8, 266. [CrossRef]

11. Choi, Y.; Zhang, N. Introduction to the Special Issue on "The Sustainable Asia Conference 2014". Sustainability 2015, 7, 1595-1602. [CrossRef]

(C) 2017 by the author; licensee MDPI, Basel, Switzerland. This article is an open access article distributed under the terms and conditions of the Creative Commons Attribution (CC BY) license (http:/ / creativecommons.org/licenses/by/4.0/). 